\title{
Error-related negativity and error awareness in a Go/No-go task
}

\begin{abstract}
Lijun Wang ${ }^{1}$, Yan Gu ${ }^{2}$, Guoxiang Zhao ${ }^{3}$ \& Antao Chen ${ }^{2 *}$
Error monitoring is crucial for the conscious error perception, however, the role of early error monitoring in error awareness remains unclear. Here, we investigated the relation between the ERN and errorrelated theta oscillations and the emergence of error awareness by conducting time- and phase-locked averaging analysis based on $4-8 \mathrm{~Hz}$ filtered data and phase-locked time frequency analysis. Results showed that while the ERN did not differ significantly between aware and unaware errors, theta power was stronger for aware errors than for unaware errors. Further, when continuous EEG was filtered outside the theta band, the ERN results confirmed this pattern. Additionally, when the non-phase-locked component was removed from continuous EEG, stronger theta power was still observed in aware errors compared to unaware errors. Collectively, these findings may suggest that (1) the ERN emerges from phase-locked component of theta band EEG activities; (2) the ERN engages in conscious error perception and serves the emerging error awareness through the activity of theta oscillations. Thus, early error monitoring is a precursor to error awareness, but this relationship is masked by high-frequency activity in aware errors when the ERN is not filtered outside the theta band in the Go/No-go task.
\end{abstract}

The ability to monitor continuously action outcomes, especially errors, is essential for executing goal-directed behaviors. Detecting and correcting current errors is one of the crucial components of error monitoring. The electroencephalography (EEG) approach, due to its high temporal resolution, is well suitable to study the time course of error monitoring. Previous studies showed that error-related negativity (ERN) and error positivity $(\mathrm{Pe})$ are specifically linked to the error monitoring ${ }^{1-3}$. The ERN, indexing early error monitoring, is a negative deflection that peaks over fronto-central scalp distribution around $50 \mathrm{~ms}$ after error responses. The ERN is thought to be generated in the medial-frontal cortex ${ }^{4-6}$. The Pe, indexing late error monitoring, is a parietal positivity following ERN that occurs at the time windows from about 200 to $500 \mathrm{~ms}$ after error responses ${ }^{3}$.

A large number of studies have investigated the neural correlates of error awareness by asking participants to subjectively report their errors, and consistently found that the late error monitoring Pe was significantly larger for aware than for unaware errors ${ }^{7-10}$. These results suggest that Pe is specifically related to the error awareness processing. However, the issue as to whether early error monitoring ERN is involved in the error awareness processing is a matter of debate. The studies employing Flanker task showed enlarged ERN amplitude for aware compared to unaware errors ${ }^{7,11,12}$, whereas this effect was not found in studies employing Stop-signal or Go/ No-go task ${ }^{13-15}$. In addition to error detection ${ }^{16}$, previous studies have demonstrated that the functional significance of ERN also reflects conflict monitoring ${ }^{17,18}$. Moreover, Di Gregorio and his colleagues (2016) utilized an error classification paradigm and found that the relationship between the ERN and error awareness was mediated by response conflict ${ }^{7}$. Thus, one possible reason for the discrepant ERN findings on error awareness across studies is the influence of the level of post-error conflict on aware errors. After the occurrence of an error, the intended correct response is still activated during the extended processing of the stimulus. Errors in the Flanker task are often due to failures of selective attention to the target or due to premature responding, thus the activated correct response may cause strong post-error conflict and enhanced ERN in aware errors. Nevertheless, in the Stop-signal/Go-Nogo task, participants are not required to press the button, and errors are mainly due to the failure of motor inhibition. In this case, post-response conflict is weak and accordingly the effect of ERN between aware and unaware errors is reduced.

Moreover, comparable ERN amplitudes for aware and unaware errors do not preclude the possibility that the ERN serves the error awareness. For instance, in the study of Hughes and Yeung ${ }^{19}$, although they reported the ERN was not different between aware and unaware errors, they indeed found that, on the single-trial level, the

${ }^{1}$ Institute of cognition, brain and health, School of Education, HeNan University, Kaifeng, 475004, China. ${ }^{2}$ Key Laboratory of Cognition and Personality of Ministry of Education, Faculty of Psychology, Southwest University, Chongqing, 400715, China. ${ }^{3}$ Faculty of Education, Henan Normal University, Xinxiang, 453007, China. *email: xscat@swu.edu.cn 


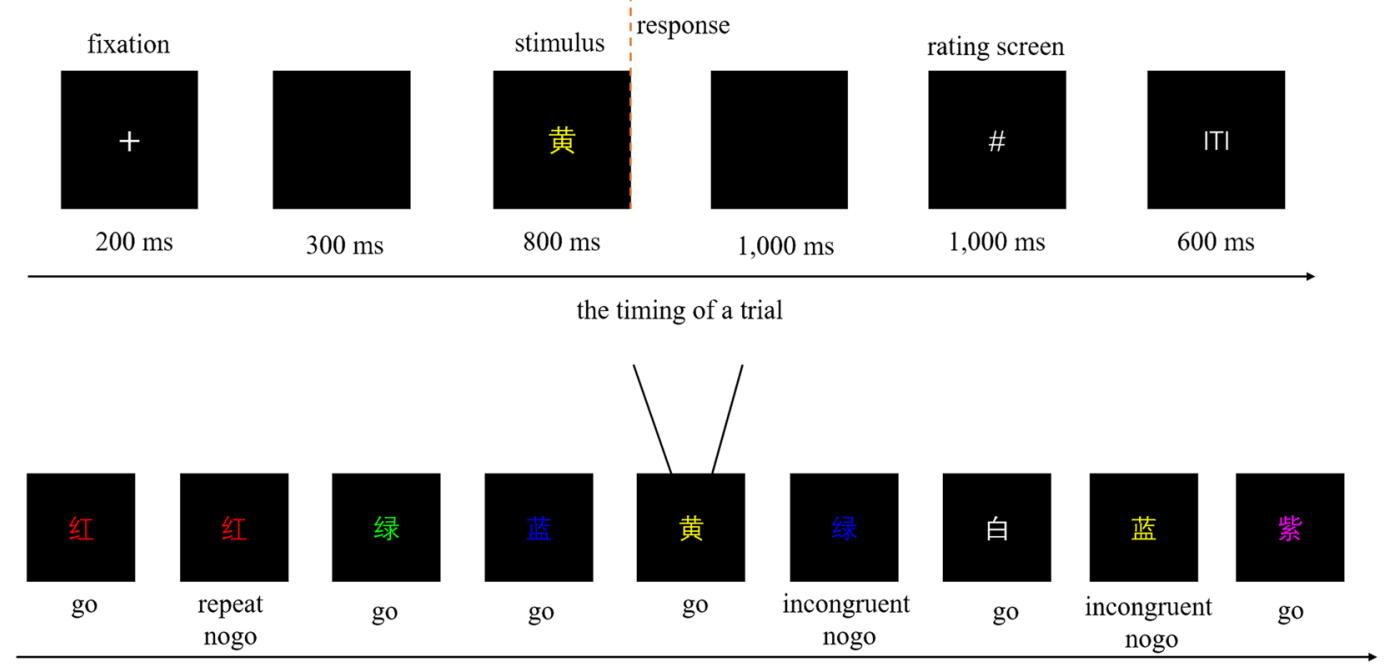

the sequence of trials

Figure 1. Schematic illustration of the procedures. The figure illustrates the timing parameters of one trial (above) and part of the sequence (below). Stimulus is terminated after Go press within $800 \mathrm{~ms}$. The hash (\#) cues participant to rate his response accuracy (error or correct) in the rating screen. ITI means intertrial interval.

more the participants consciously perceived their errors, the larger the ERN. Therefore, it is appealing to investigate whether the ERN reflects error awareness by adopting sensitive analyzing methods.

Several studies have demonstrated that the ERN is associated with the phase resetting of frontal theta band $(4-8 \mathrm{~Hz})^{20,21}$. Crucially, theta band has been considered as an effective indicator of conscious error perception $^{12,22}$. In the present study, the time- and phase-locked averaging analysis based on $4-8 \mathrm{~Hz}$ filtered data and the phase-locked time frequency analysis were conducted to examine whether the early error monitoring engaged in the error awareness processing.

Specifically, an error awareness task ${ }^{23}$ based on a Go/No-go task (Fig. 1) was employed to study the above issue. Considering that the usage of an error signal button might lead to a response bias toward signaling (participants might signal their correct responses as errors, increasing the false alarm rates) or not signaling an error (the measurement of unaware errors might be contaminated by the potentially conscious error trials $)^{24}$, we instructed participants to make a response to indicate perceived response accuracy in both error and correct cases during rating screen ${ }^{7,10,25}$. If an error was rated as error response, the corresponding trial would be defined as aware error; if an error was reported as correct response, the trial would be defined as unaware error.

Based on the functional role of theta band, if the early stage immediately following an error completed the conscious error perception and served the error awareness processing in the Go/No-go task, the ERN in the $4-8 \mathrm{~Hz}$ filter band (theta-ERN) were expected to be significantly larger for aware than for unaware errors, and the oscillations in the theta band were expected to be significantly larger for aware than for unaware errors.

\section{Results}

All trials were sorted into three categories on the basis of task response: correct go, aware error and unaware error. When a participant responded correctly on a go trial, this trial was termed as a correct go; when a participant correctly identified that he/she responded mistakenly on a No-go trial, this trial was termed as an aware error; when a participant classified his response to a No-go trial as a correct go trial (it was really an error No-go trial), this trial was termed as an unaware error.

In order to yield sufficient numbers of aware and unaware errors, we followed previous studies ${ }^{23,26}$ to instruct participants to withhold their responses in two circumstances. The first is when a word was presented on two consecutive trials (repeat No-go trials) and the second is when font color of the word and its meaning were inconsistent (incongruent No-go trials). Moreover, to warrant reliability of statistical analysis, we analyzed aware and unaware errors by merging two No-go types ${ }^{23,26}$.

Effects of variables with more than two levels were tested by analysis of variance (ANOVA) with repeated measurement. To compensate for violations of sphericity, Greenhouse-Geisser correction was employed where appropriate, and corrected $p$ values (but uncorrected degrees of freedom) were reported. Differences between conditions were tested by Pairwise Comparisons using two-tailed $t$ tests ${ }^{7}$.

Behavioral results. Participants correctly withheld their responses on $51 \%$ of No-go trials, with significantly better performance for incongruent No-go than for repeat No-go trials ( $54 \mathrm{vs.} 43 \%), t(30)=2.85, p=0.008$, Cohen's $d=0.51$. And participants reported being aware of $81 \%$ of all commission errors, with $70 \%$ of aware errors occurring on incongruent no-go trials. The mean available number of aware errors and unaware errors was 85 and 24 respectively. The RT of correct go $(477 \pm 12 \mathrm{~ms})$ was significantly slower than aware $(444 \pm 11 \mathrm{~ms}$, $t(30)=8.57, p<0.001$, Cohen's $\mathrm{d}=1.54)$ and unaware error $(452 \pm 17 \mathrm{~ms}, t(30)=3.10, p=0.004$, Cohen's 


\begin{tabular}{|c|c|c|c|c|c|c|c|c|c|c|c|c|c|c|c|}
\hline \multirow[b]{2}{*}{ participant } & \multicolumn{3}{|c|}{ ERN $(\mu v)$} & \multicolumn{3}{|l|}{$\operatorname{Pe}(\mu \mathrm{v})$} & \multicolumn{3}{|c|}{ theta-ERN $(\mu v)$} & \multicolumn{3}{|c|}{ alpha-ERN $(\mu v)$} & \multicolumn{3}{|c|}{ beta-ERN $(\mu \mathrm{v})$} \\
\hline & $\begin{array}{l}\text { aware } \\
\text { error }\end{array}$ & $\begin{array}{l}\text { unaware } \\
\text { error }\end{array}$ & $\begin{array}{l}\text { correct } \\
\text { go }\end{array}$ & $\begin{array}{l}\text { aware } \\
\text { error }\end{array}$ & \begin{tabular}{|l} 
unaware \\
error
\end{tabular} & \begin{tabular}{|l} 
correct \\
go
\end{tabular} & $\begin{array}{l}\text { aware } \\
\text { error }\end{array}$ & \begin{tabular}{|l|} 
unaware \\
error
\end{tabular} & \begin{tabular}{|l|} 
correct \\
go
\end{tabular} & $\begin{array}{l}\text { aware } \\
\text { error }\end{array}$ & $\begin{array}{l}\text { unaware } \\
\text { error }\end{array}$ & \begin{tabular}{|l|} 
correct \\
go
\end{tabular} & $\begin{array}{l}\text { aware } \\
\text { error }\end{array}$ & $\begin{array}{l}\text { unaware } \\
\text { error }\end{array}$ & $\begin{array}{l}\text { correct } \\
\text { go }\end{array}$ \\
\hline 1 & -1.39 & 0.67 & 0.74 & -5.97 & -7.27 & -11.14 & -2.02 & -0.64 & -0.16 & -1.08 & 1.46 & 1.59 & -0.31 & 1.98 & 1.55 \\
\hline 2 & -0.29 & -1.59 & 0.30 & 6.71 & 1.54 & -7.17 & -0.57 & -1.23 & -0.30 & -0.12 & -2.06 & -0.31 & -0.08 & -2.54 & -0.37 \\
\hline 3 & -4.55 & -2.13 & -3.72 & -2.37 & -4.62 & -7.84 & -2.50 & -1.18 & -1.93 & -4.19 & -1.03 & -3.19 & -4.29 & -0.31 & -3.32 \\
\hline 4 & -0.61 & -0.89 & -1.18 & 4.97 & 2.43 & 0.30 & -0.93 & -0.95 & -0.94 & -0.05 & -0.17 & -0.07 & -0.56 & -0.82 & -0.58 \\
\hline 5 & -0.30 & -0.19 & 1.63 & 10.51 & 5.06 & 4.74 & -0.65 & -0.32 & 0.86 & -0.13 & 1.52 & 2.58 & -0.22 & 1.50 & 2.63 \\
\hline 6 & 5.05 & -0.41 & 2.29 & 3.10 & -2.22 & -5.99 & -2.73 & -1.30 & -3.75 & 5.65 & 1.48 & 3.01 & 5.03 & 1.59 & 3.14 \\
\hline 7 & 0.41 & -1.74 & 1.66 & -0.21 & -1.60 & -2.59 & 0.81 & -2.48 & -1.34 & 0.50 & -1.25 & 2.23 & 0.46 & -0.55 & 2.22 \\
\hline 8 & -1.61 & -0.87 & -0.22 & 3.33 & 3.39 & 1.55 & -1.31 & -1.35 & -0.45 & -1.38 & -0.84 & -0.05 & -0.98 & -0.62 & -0.15 \\
\hline 9 & -0.96 & -5.54 & 2.00 & 9.18 & -1.95 & -0.42 & -1.04 & -2.13 & -0.76 & -0.48 & -3.98 & 2.46 & -0.28 & -4.15 & 2.52 \\
\hline 10 & 0.54 & -1.41 & 0.10 & 7.16 & -1.87 & -1.50 & 0.10 & -0.95 & 0.80 & 1.25 & -0.26 & 0.76 & 1.34 & -0.25 & 0.66 \\
\hline 11 & -0.62 & -0.23 & 2.30 & 5.09 & 2.55 & -1.55 & -1.06 & -0.57 & 0.23 & 0.03 & 0.78 & 3.30 & -0.30 & 0.37 & 3.30 \\
\hline 12 & -3.03 & -2.20 & 0.25 & 2.79 & -0.79 & -4.62 & -0.67 & -0.72 & 0.57 & -2.46 & -1.12 & 1.18 & -2.51 & -1.28 & 1.12 \\
\hline 13 & 1.78 & 0.32 & 0.73 & 2.44 & -2.45 & -5.66 & 2.53 & 1.71 & 1.96 & 2.45 & 1.22 & 1.50 & 2.65 & 0.67 & 1.39 \\
\hline 14 & 0.10 & -0.57 & -0.11 & 10.55 & 5.76 & 1.69 & -1.48 & -0.95 & -0.69 & 0.00 & 0.66 & 0.69 & 0.31 & 0.08 & 0.73 \\
\hline 15 & -1.33 & -4.09 & 0.49 & 7.89 & -3.34 & -3.28 & -0.39 & -0.08 & 0.28 & -0.48 & -3.14 & 0.85 & -0.76 & -3.19 & 0.89 \\
\hline 16 & -1.41 & 1.85 & 3.48 & 9.43 & 4.40 & 2.03 & -0.50 & 0.87 & 1.74 & -0.84 & 2.91 & 4.56 & -1.16 & 2.98 & 4.57 \\
\hline 17 & -0.43 & -0.23 & 1.57 & 2.40 & -2.29 & -1.75 & -1.61 & -0.98 & 0.12 & 0.08 & 0.10 & 2.42 & -0.54 & -0.18 & 2.41 \\
\hline 18 & -5.58 & -2.68 & -15.01 & -2.59 & -11.59 & -22.91 & -2.27 & -0.63 & -0.39 & -5.40 & -3.19 & -15.29 & -5.64 & -3.43 & -15.17 \\
\hline 19 & -4.01 & -0.02 & 0.95 & 4.88 & 0.30 & -2.84 & -1.10 & -0.40 & -0.12 & -3.35 & 1.63 & 2.55 & -3.67 & 2.00 & 2.54 \\
\hline 20 & -3.07 & -0.98 & -2.50 & -4.38 & -6.37 & -5.13 & -0.41 & 1.24 & -0.06 & -2.68 & -0.85 & -1.79 & -2.82 & -0.41 & -1.83 \\
\hline 21 & -6.64 & -1.49 & 0.31 & 6.29 & 6.59 & 1.38 & -1.02 & -0.41 & 1.21 & -5.83 & -0.35 & 0.95 & -6.58 & 0.16 & 0.90 \\
\hline 22 & 1.63 & -1.81 & 0.57 & 10.45 & 6.82 & -1.02 & 1.24 & 1.91 & 0.93 & 2.21 & -0.71 & 1.24 & 1.94 & 0.77 & 1.03 \\
\hline 23 & -1.73 & -1.41 & 2.02 & 8.05 & 1.10 & -2.22 & -1.07 & -0.77 & 1.13 & -1.01 & -1.40 & 2.99 & -1.39 & -1.95 & 2.99 \\
\hline 24 & -1.64 & 0.28 & 0.28 & 4.67 & -2.56 & -3.42 & -1.85 & -0.73 & -1.82 & -1.02 & 1.18 & 1.89 & -0.88 & 1.48 & 1.92 \\
\hline 25 & -0.08 & -1.25 & 3.06 & 4.59 & -2.94 & -2.34 & -2.11 & -2.95 & 0.23 & 0.42 & -0.69 & 3.33 & 0.28 & -0.55 & 3.34 \\
\hline 26 & -6.23 & -4.29 & -1.98 & 4.18 & -1.80 & -5.45 & -5.35 & -3.72 & -2.32 & -5.23 & -4.39 & -1.73 & -6.00 & -5.33 & -1.66 \\
\hline 27 & 5.03 & 2.45 & 4.67 & -8.19 & -4.80 & -11.43 & -4.22 & -0.91 & -1.73 & 5.59 & 3.03 & 5.78 & 5.86 & 2.75 & 5.62 \\
\hline 28 & -2.10 & 0.16 & 2.15 & 4.56 & -2.55 & -2.50 & 0.02 & -0.01 & 0.80 & -0.92 & 1.19 & 2.20 & -1.31 & 1.70 & 2.16 \\
\hline 29 & -3.48 & -1.41 & -1.33 & 4.98 & 0.81 & -2.15 & -2.43 & -0.37 & -0.21 & -3.17 & -0.44 & -0.43 & -2.89 & -1.09 & -0.44 \\
\hline 30 & -4.50 & -1.96 & 0.74 & 1.81 & -2.31 & -2.52 & -1.91 & -0.94 & 0.56 & -3.60 & -1.04 & 1.62 & -3.81 & -1.52 & 1.57 \\
\hline 31 & 0.02 & -0.22 & 1.09 & 2.27 & 0.32 & -5.31 & -0.21 & 0.31 & 0.98 & 0.79 & 0.71 & 2.70 & 0.92 & 0.57 & 2.70 \\
\hline mean & -1.32 & -1.09 & 0.24 & 3.83 & -0.72 & -3.58 & -1.18 & -0.70 & -0.15 & -0.79 & -0.29 & 0.95 & -0.91 & -0.31 & 0.92 \\
\hline
\end{tabular}

Table 1. The individual performances on the ERN, Pe, theta-ERN, alpha-ERN and beta-ERN.

$\mathrm{d}=0.56$ ). However, the RT was not different between aware and unaware error, $t(30)=0.92, p=0.36$, Cohen's $\mathrm{d}=0.17$.

ERP results. As suggested by a number of researchers ${ }^{27,28}, 10$ to 15 available trials are required for a reliable error processing. Therefore, only those who had at least 10 available trials for each error type were included in the analysis. The mean available number of aware and unaware errors was 76 and 22 respectively. And the individual performances on the ERN, Pe, theta-ERN, alpha-ERN and beta-ERN were listed in Table 1.

An ANOVA on the ERN for three trial types showed a significant effect of trial type, $F(2,60)=5.30, p=0.009$, $\eta^{2}=0.15$ (Fig. 2a). Pairwise Comparisons (Fisher, LSD) revealed that the ERN was significantly larger for both error types than correct go trials (correct go: $0.24 \pm 0.60 \mu \mathrm{v}$; aware error vs. correct go: $t(30)=-2.92, p=0.007$, Cohen's $\mathrm{d}=-0.53$; unaware error vs. correct go: $t(30)=-2.38, p=0.024$, Cohen's $\mathrm{d}=0.43)$. However, the ERN showed no difference between aware $(-1.32 \pm 0.49 \mu \mathrm{v})$ and unaware errors $(-1.09 \pm 0.29 \mu \mathrm{v}), t(30)=-0.51$, $p=0.61$, Cohen's $\mathrm{d}=-0.09$.

The ANOVA on Pe showed that the effect of trial type was significant, $F(2,60)=76.199, p<0.001$, $\eta^{2}=0.72$ (Fig. 2b). Pairwise Comparisons (Fisher, LSD) revealed that the Pe was significantly larger for aware $(3.83 \pm 0.85 \mu \mathrm{v})$ than for unaware errors $(-0.72 \pm 0.75 \mu \mathrm{v} ; t(30)=7.78, p<0.001$, Cohen's $\mathrm{d}=1.40)$ and correct go trials $(-3.58 \pm 0.91 \mu \mathrm{v} ; t(30)=10.90, p<0.001$, Cohen's $\mathrm{d}=1.96)$. Moreover, the Pe was significantly larger for unaware errors than for correct go trials, $t(30)=5.27, p<0.001$, Cohen's $\mathrm{d}=0.95$.

Importantly, the results of ANOVA on theta-ERN showed that the effect of trial type was significant, $F(2$, $60)=12.32, p<0.001, \eta^{2}=0.29$ (Fig. 3a). Pairwise Comparisons (Fisher, LSD) revealed that theta-ERN was significantly larger for aware $(-1.18 \pm 0.27 \mu \mathrm{v})$ than for unaware errors $(-0.70 \pm 0.21 \mu \mathrm{v} ; t(30)=-2.26, p=0.032$, Cohen's $\mathrm{d}=-0.41)$ and correct go trials $(-0.15 \pm 0.22 \mu \mathrm{v} ; t(30)=-4.95, p<0.001$, Cohen's $\mathrm{d}=-0.90)$. Notably, theta-ERN was significantly larger for unaware errors than for correct go trials, $t(30)=-2.73, p=0.01$, Cohen's $\mathrm{d}=-0.5$. 
a

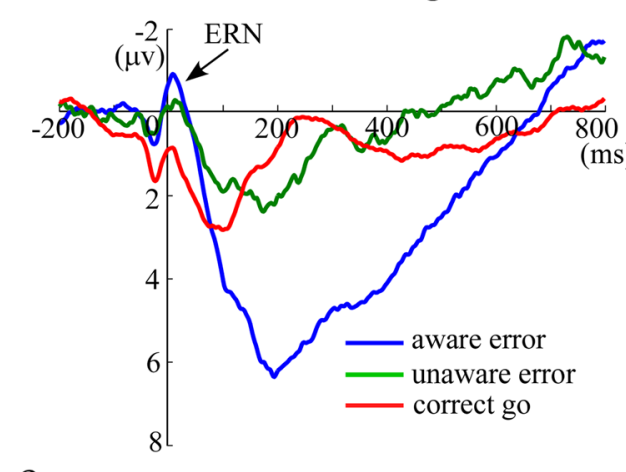

$\mathrm{c}$

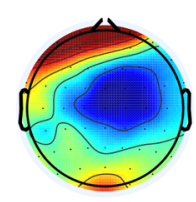

aware-correct fronto-central region

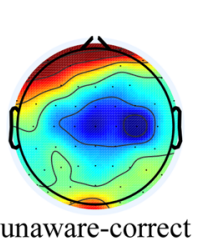

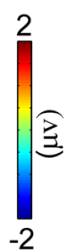

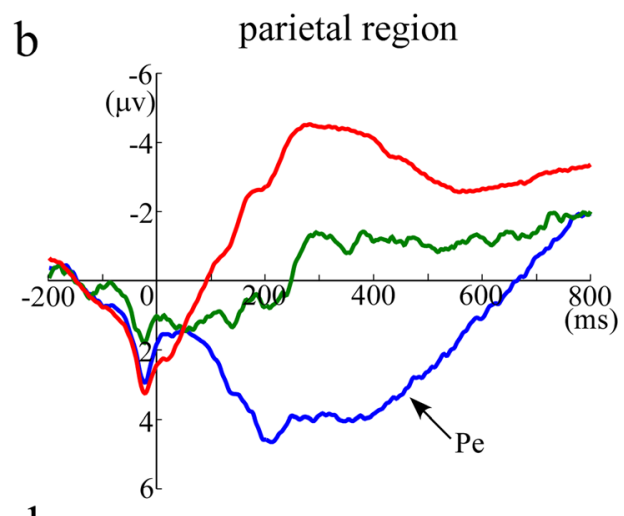

d

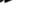

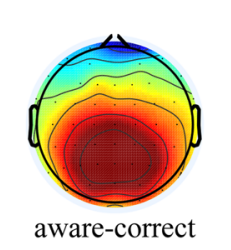

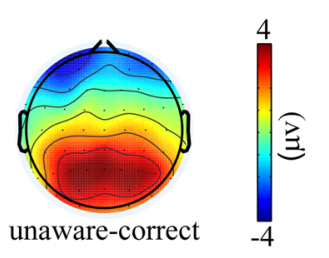

Figure 2. The ERN and Pe results in the present study. Panel a shows the response-locked grand-averaged ERP waveforms for the ERN (-20 to $50 \mathrm{~ms})$ in the fronto-central region $[(\mathrm{FCz}+\mathrm{FC} 1+\mathrm{FC} 2+\mathrm{Cz}+\mathrm{C} 1+\mathrm{C} 2) / 6]$. Panel $b$ shows the response-locked grand-averaged ERP waveforms for the Pe (150 to $500 \mathrm{~ms})$ in the parietal region $[(\mathrm{CPz}+\mathrm{CP} 1+\mathrm{CP} 2+\mathrm{Pz}+\mathrm{P} 1+\mathrm{P} 2) / 6]$. Data were filtered offline with a passband $0.1-30 \mathrm{~Hz}$. Blue line indicates the neural activity of aware errors, green line indicates the neural activity of unaware errors, and red line indicates the neural activity of correct go trials. Panels $\mathrm{c}$ shows the difference topography distribution of ERN between aware errors and correct go trials and the difference topography distribution of ERN between unaware errors and correct go trials, respectively. Panel d shows the difference topography distribution of Pe between aware errors and correct go trials and the difference topography distribution of Pe between unaware errors and correct go trials, respectively.

The results of ANOVA on alpha-ERN showed that the effect of trial type was significant, $F(2,60)=6.15$, $p=0.005, \eta^{2}=0.17$ (Fig. 3b). Pairwise Comparisons (Fisher, LSD) revealed that alpha-ERN was significantly larger for both error types than correct go trials $(0.95 \pm 0.63 \mu \mathrm{v}$; aware error vs. correct go: $t(30)=-3.20$, $p=0.003$, Cohen's $\mathrm{d}=-0.58$; unaware error vs. correct go: $t(30)=-2.30, p=0.028$, Cohen's $\mathrm{d}=-0.41)$. However, the alpha-ERN was not different between aware $(-0.79 \pm 0.49 \mu \mathrm{v})$ and unaware errors $(-0.29 \pm 0.32$ $\mu \mathrm{v}), t(30)=-1.13, p=0.267$, Cohen's $\mathrm{d}=-0.20$.

The results of ANOVA on beta-ERN showed that the effect of trial type was significant, $F(2,60)=6.22$, $p=0.004, \eta^{2}=0.17$ (Fig. 3c). Pairwise Comparisons (Fisher, LSD) revealed that alpha-ERN was significantly larger for both error types than correct go trials $(0.92 \pm 0.63 \mu \mathrm{v}$; aware error vs. correct go: $t(30)=-3.27$, $p=0.003$, Cohen's $\mathrm{d}=-0.59$; unaware error vs. correct go: $t(30)=-2.22, p=0.034$, Cohen's $\mathrm{d}=-0.41)$. However, the alpha-ERN revealed no difference between aware $(-0.91 \pm 0.50 \mu \mathrm{v})$ and unaware errors $(-0.31 \pm 0.35 \mu \mathrm{v})$, $t(30)=-1.29, p=0.209$, Cohen's $\mathrm{d}=-0.23$.

Time-frequency results. The mean available trial number of aware and unaware errors was 70 and 22 respectively. And the individual performances of ERSP on the theta, alpha and phase-locked theta bands were listed in Table 2. The modulation associated with error awareness mainly occurred in the fronto-central and occipito-parietal regions, which were illustrated in Fig. 4 . In the above S-ROIs, the TF-ROIs theta $(4-7 \mathrm{~Hz}$, -150 to $200 \mathrm{~ms})$ and alpha $(8-14 \mathrm{~Hz}, 200$ to $600 \mathrm{~ms})$ that showed the most pronounced task-related effects were defined (in rectangles in Fig. $4 \mathrm{a}, p<0.05$, FDR corrected). The ERSP magnitudes within defined S-ROIs for aware and unaware errors were entered into the paired-samples $t$ test (Fig. 4c). For the fronto-central region, the result showed that theta power was significantly larger for aware $(17.29 \pm 3.85 \mathrm{ER} \%)$ than for unaware errors $(5.78 \pm 3.24 \mathrm{ER} \%), t(30)=6.15, p<0.001$, Cohen's $\mathrm{d}=1.10$. For the occipito-parietal region, the result showed that alpha power was significantly smaller for aware $(-10.58 \pm 2.87 \mathrm{ER} \%)$ than for unaware errors $(3.61 \pm 3.47$ ER\%), $t(30)=-4.073, p<0.001$, Cohen's $\mathrm{d}=-0.73$.

Further, we examined theta activity defined in the fronto-central region by removing the non-phased-locked component from continuous EEG, the result revealed that theta power of aware errors $(127.93 \pm 16.04$ ER\%) was still significantly larger compared with unaware errors $(31.00 \pm 9.08 \mathrm{ER} \%), t(30)=5.74, p<0.001$, Cohen's $\mathrm{d}=1.03$ (Fig. 5). 
(a)

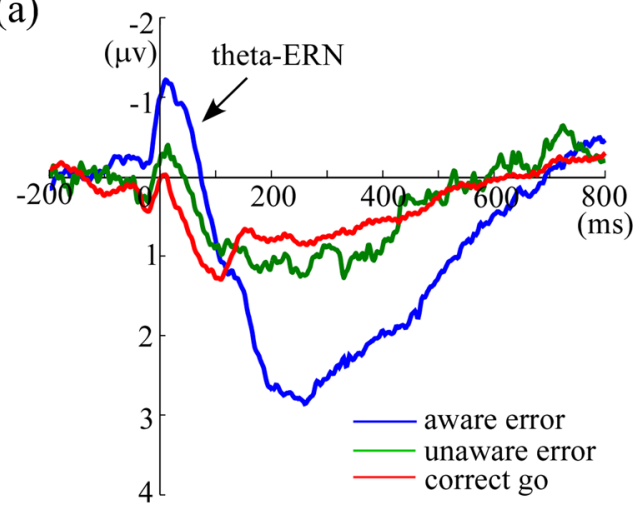

(b)

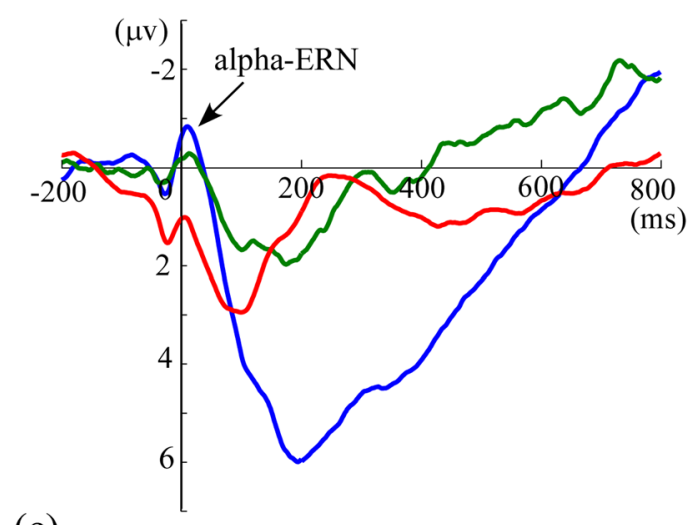

(c)

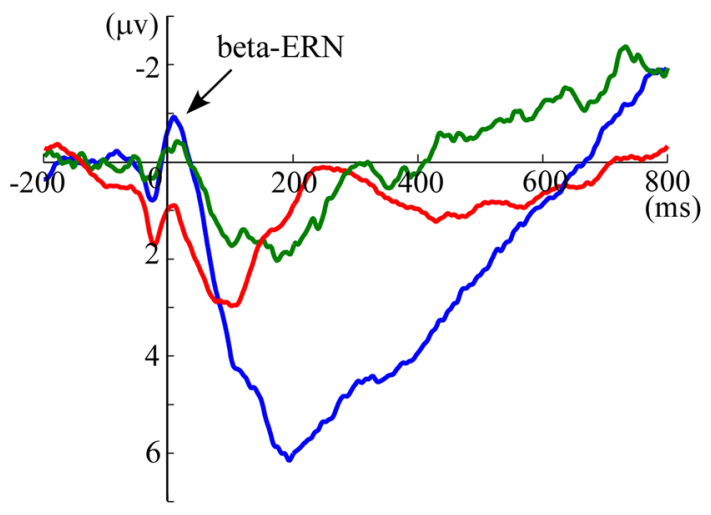

Figure 3. The ERN results in the theta, alpha and beta frequency bands, respectively. Panel a shows theta-ERN results, indicating that theta-ERN is significantly larger for aware compared with unaware errors. Panels $b$ and c show alpha-ERN and beta-ERN results, indicating that alpha-ERN and beta-ERN are both not difference between aware and unaware errors. Blue line indicates the neural activity of aware errors, green line indicates the neural activity of unaware errors, and red line indicates the neural activity of correct go trials.

\section{Discussion}

The goal of the present study was to investigate whether the early error monitoring engaged in the emergence of error awareness in the Go/No-go task. Considering the ERN is sensitive to the theta frequency $(4-8 \mathrm{~Hz})^{20,29}$, in addition to the traditional ERP and time-frequency analyses, the ERP analysis based on $4-8 \mathrm{~Hz}$ filtered data and the phase-locked time-frequency analysis were conducted to examine the above issue. The results showed that the ERN was comparable between aware and unaware errors, but theta-ERN was significantly larger for aware than for unaware errors. Moreover, increased theta power was observed in aware compared with unaware errors, even when the non-phased-locked component was removed from continuous EEG. In addition, increased Pe amplitude and enhanced alpha power was observed in aware compared with unaware errors. These findings may suggest that the early error monitoring engages in conscious error perception and serves the error awareness processing in the Go/No-go task.

Traditional ERP measurements showed that the ERN did not differ between aware and unaware errors, paralleling with previous studies ${ }^{9,15,25}$. In the present study, when the ERN was examined in the theta $(4-8 \mathrm{~Hz})$, alpha 


\begin{tabular}{|c|c|c|c|c|c|c|}
\hline \multirow[b]{2}{*}{ participant } & \multicolumn{2}{|c|}{ Theta (\%ER) } & \multicolumn{2}{|l|}{ Alpha (\%ER) } & \multicolumn{2}{|c|}{ Phase-locked theta (\%ER) } \\
\hline & \begin{tabular}{|l}
$\begin{array}{l}\text { aware } \\
\text { error }\end{array}$ \\
\end{tabular} & unaware error & aware error & unaware error & $\begin{array}{l}\text { aware } \\
\text { error }\end{array}$ & unaware error \\
\hline 1 & 36 & 24 & 7 & 14 & 272 & 110 \\
\hline 2 & 20 & 12 & -13 & 34 & 192 & 4 \\
\hline 3 & 26 & 13 & -15 & 8 & 231 & 13 \\
\hline 4 & 13 & 18 & -5 & 6 & 215 & 31 \\
\hline 5 & -1 & -7 & -12 & 15 & 154 & 26 \\
\hline 6 & 7 & -23 & 13 & 11 & 60 & -20 \\
\hline 7 & 2 & 7 & -21 & -8 & 99 & 175 \\
\hline 8 & -4 & -15 & -10 & -21 & 81 & -17 \\
\hline 9 & -6 & -1 & -26 & 1 & 58 & -13 \\
\hline 10 & 14 & 4 & -7 & 1 & 227 & 5 \\
\hline 11 & 16 & 8 & -15 & 15 & 91 & -8 \\
\hline 12 & -8 & -7 & -12 & 11 & 51 & -3 \\
\hline 13 & 21 & 6 & -14 & -7 & 41 & 8 \\
\hline 14 & 20 & 1 & -4 & -11 & 126 & 25 \\
\hline 15 & 8 & -10 & -10 & 6 & 98 & 46 \\
\hline 16 & 8 & -7 & -2 & 2 & 33 & 9 \\
\hline 17 & -5 & -23 & -22 & -7 & 39 & -18 \\
\hline 18 & 30 & 32 & 6 & 35 & 170 & 139 \\
\hline 19 & 13 & 3 & -14 & 64 & 111 & 29 \\
\hline 20 & 21 & 8 & -9 & -6 & 169 & 54 \\
\hline 21 & 3 & -12 & -51 & -36 & 25 & 0 \\
\hline 22 & 3 & -2 & -43 & -41 & 35 & 38 \\
\hline 23 & 9 & 10 & -22 & 0 & 47 & 9 \\
\hline 24 & 4 & 0 & -17 & 4 & 68 & 59 \\
\hline 25 & 58 & 47 & -16 & 12 & 319 & -27 \\
\hline 26 & 35 & 6 & 1 & -5 & 207 & 44 \\
\hline 27 & 73 & 44 & 22 & 10 & 355 & 20 \\
\hline 28 & 22 & -0.8 & -1 & -3 & 101 & 61 \\
\hline 29 & -3 & -7 & -19 & 7 & 38 & 6 \\
\hline 30 & 80 & 48 & 27 & 3 & 172 & 151 \\
\hline 31 & 21 & 3 & -24 & -2 & 81 & 5 \\
\hline mean & \begin{tabular}{|l|}
17.29 \\
\end{tabular} & 5.78 & -10.58 & 3.61 & $\mid 127.93$ & 31 \\
\hline
\end{tabular}

Table 2. The individual performances of ERSP on the theta, alpha and phase-locked theta.

$(8-14 \mathrm{~Hz})$ and beta $(14-30 \mathrm{~Hz})$ frequency bands respectively, significantly enlarged ERN for aware than unaware errors was observed when the continuous EEG was filtered in the theta frequency band, but not in the alpha and beta frequency bands. Thus, these results provides further evidence that the ERN emerges from phase locking of theta band EEG activity ${ }^{21,30}$. Moreover, these results suggest that early error monitoring completes conscious error perception after aware errors in the Go/No-go task. Although No-go trials exerts global suppression effect on the motor system, weak post-error conflict might result in attenuated post-response inhibition control. It has been suggested that the high frequency band (such as alpha and beta bands) reflects the function of inhibition control $^{31,32}$. Under this circumstances, the EEG activities from high frequency bands might mask the ERN difference between aware and unaware errors in the traditional analysis. Thus, a larger ERN amplitude was observed for aware than for unaware errors when the high frequency band EEG activities were filtered.

Consistent with the aforementioned theta-ERN finding, the theta power of aware errors was significantly stronger compared with unaware errors. Previous study explained the discrepancy between the ERN and theta power as the majority of theta activities caused by aware errors were not phase-locked to the error response ${ }^{12}$. This explanation seems plausible given that Trujillo and Allen ${ }^{33}$ found that, following errors, the increase in non-phase-locked power was larger than phase-locked power. To examine this potential confusion, we further analyzed the theta activity by removing the non-phase-locked component from continuous EEG. Importantly, stronger theta power was still observed for aware than for unaware error. Theta oscillation is always associated with error perception and the need of enhanced control ${ }^{22,29,30,34}$. Thus, aware errors inducing stronger power may imply that error information signaled performance system to recruit more cognitive resources to adjust error behaviors.

Concerning the Pe, the activity was significantly larger for aware compared with unaware errors. The functional significance of Pe has been associated with the error awareness ${ }^{7,15,25}$. In particular, some studies have demonstrated that Pe reflects the strength of evidence that an error has occurred ${ }^{10,35,36}$. If the error evidences from multiple sources reach the perceivable level, the error is more likely to be reported. If not, the error will 


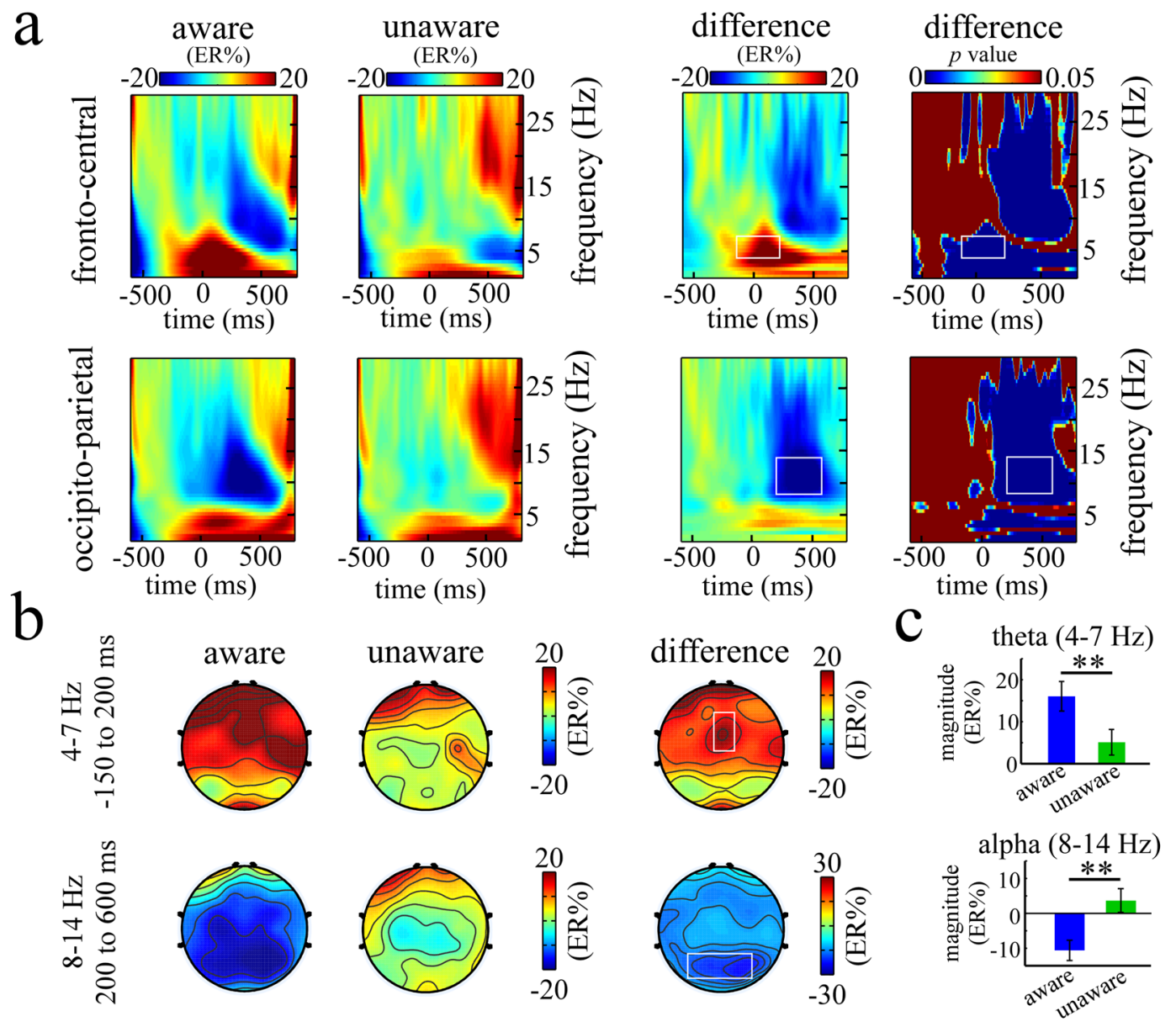

Figure 4. The time-frequency results for aware and unaware errors during the response period. Panel a shows the grand-average time-frequency representations (expressed as ER\%) for aware and unaware errors and the difference time-frequency representations (aware errors minus unaware errors) within the defined S-ROIs, including fronto-central region $[(\mathrm{Fz}+\mathrm{FCz}+\mathrm{Cz}) / 3]$ and occipito-parietal region $[(\mathrm{Pz}+\mathrm{P} 3+\mathrm{P} 4+\mathrm{POz}+\mathrm{PO} 3+\mathrm{PO} 4) / 6]$. The corresponding difference $\mathrm{p}$ map is the result of bootstrapping statistical analysis at the significance level of $\mathrm{p}<0.05$ (FDR corrected), which is used to define the TF-ROI in each S-ROI. Note that a pre-response interval from -600 to $-100 \mathrm{~ms}$ is used as the baseline. The timefrequency pixels displaying a significant difference from the baseline are colored in blue. The significant task-related TF-ROIs are outlined in the rectangles. Each row corresponds to one S-ROI corresponding to the largest modulation of the task-related effects. X-axis, time (ms); Y-axis, frequency ( $\mathrm{Hz})$. Panel b shows the scalp topographies of ERSP magnitudes for aware and unaware errors and the difference topographies (aware errors minus unaware errors) within the defined TF-ROIs (theta band: $4-7 \mathrm{~Hz},-150$ to $200 \mathrm{~ms}$; alpha band: $8-14 \mathrm{~Hz}$, 200 to $600 \mathrm{~ms}$ ). The significant task-related S-ROIs are outlined in the white rectangles. Panel c shows mean ERSP magnitudes (expressed as ER\%) for aware and unaware errors in the theta and alpha bands, respectively.

not be subjectively reported. Thus, relatively smaller Pe was induced by unaware errors might suggest that error information from the other sources was still coded in unaware errors although the occurrence of an error was not successfully perceived.

Similarly, stronger alpha power was observed for aware than for unaware errors. The study in the Cohen, Simon, and Lamme ${ }^{37}$ utilized spectral granger causality and found that bottom-up directional synchrony (from occipital to prefrontal) mainly occurred in the alpha band. This finding may suggest alpha band in the occipito-parietal region can obtain the information of response outcome from sensory systems and motor systems in a bottom-up way, supporting the view that late error monitoring accumulates error evidences.

Notably, several studies have demonstrated that error monitoring includes two fundamental stages, information input and result output ${ }^{10,35,36}$. Combined findings from the theta-ERN and theta oscillation suggest that early error monitoring is responsible for registering error information immediately after error commission and initiating the executive control. Thus, the early error monitoring is involved in the preparation of error awareness. While the findings from the Pe and alpha oscillation suggest that late error monitoring is responsible for accumulating the error information from various sources and triggering the emergence of error awareness. Accumulating error information is a time-consuming process. This could explain, why previous studies consistently demonstrated late error monitoring rather than early error monitoring was the precursor of error awareness. 

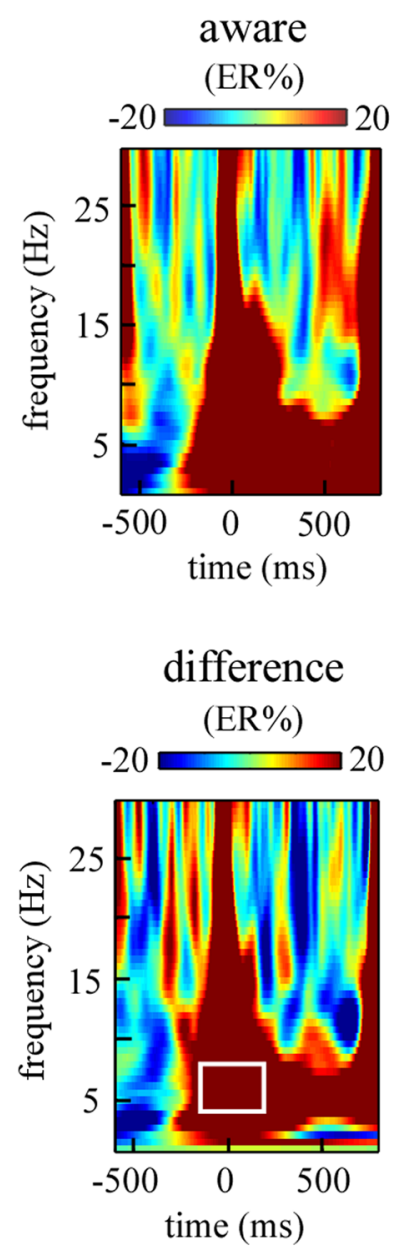

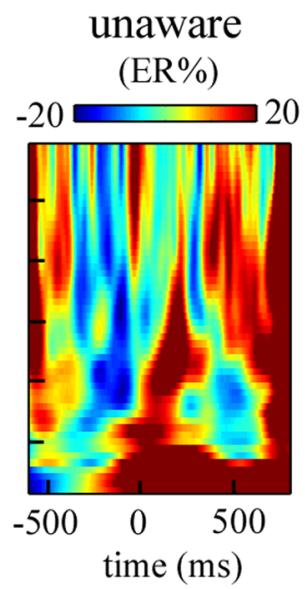

\section{difference}

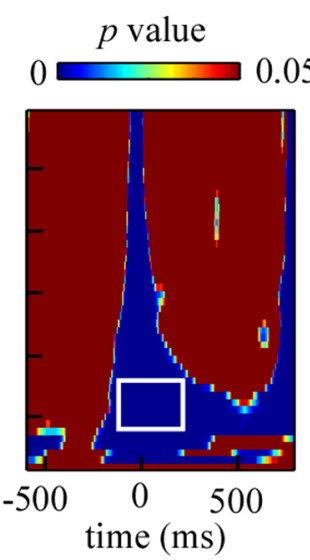

Figure 5. The phase-locked activity of theta band defined in fronto-central region. This figure shows the phase-locked time-frequency representations (expressed as ER\%) for aware and unaware errors and the difference time-frequency representations between aware and unaware errors within the fronto-central region $[(\mathrm{Fz}+\mathrm{FCz}+\mathrm{Cz}) / 3]$.

However, some limitations of the current study are worth being noted. First, No-go trials include repeat No-go and incongruent No-go. The former engages working memory processing, in which participants need to compare the current stimulus and the previous stimulus. The latter requires participants to conduct a psychological processing associated with Stroop task effect, in which participants need to evaluate stimulus congruency. However, previous studies have found that both ERN and theta power can be influenced and differently modulated by task-related features ${ }^{7,38,39}$ like working memory load ${ }^{40}$ and stimulus congruency ${ }^{18}$. To address this question, we tried to analyze the aware and unaware errors in repeat No-go and incongruent No-go errors. Unfortunately, there are insufficient unaware errors to warrant statistical comparison. Future studies might utilize other task paradigm such as Stop-signal task to verify current findings. Second, the study of Fisher et al. demonstrated that the number of errors committed per participant negatively correlates with ERN magnitude ${ }^{41}$. In the present study, the available trial number of aware errors was more than that of unaware errors. However, the theta-ERN was still larger for aware than unaware errors. Thus, error trial-number differences between aware and unaware errors may not impact the pattern of current result.

\section{Conclusion}

The present study demonstrated that when the continuous EEG was filtered outside the theta band, a significantly enlarged theta-ERN was observed for aware compared to unaware errors. Moreover, theta power was stronger for aware than for unaware errors, even when the non-phase locked components were removed from continuous EEG. Taken together, our findings suggested that early error monitoring might execute conscious error perception and serve the emergence of error awareness though the expression of theta oscillations in the Go/No-go task.

\section{Materials and Methods}

Participants. Thirty-six healthy, right-handed volunteers (22 females, 19-26 years old) were recruited to take part in the experiment for payment. All participants had normal color perception, and normal or corrected-to-normal vision. Data from five participants were removed due to the bad EEG record (too many artifacts) 
or bad behavioral performance. Finally, the data from thirty-one participants ( 18 females) were included in the behavioral and EEG analysis. All participants provided written informed consent before the experiment and all of them were naive to the purpose of the experiment. The study was in accordance with the Declaration of the Southwest University (SWU) Brain Imaging Center Institutional Review Board and approved by the ethics committee of SWU.

Apparatus and task. The experiment was conducted on a 17-inch monitor of a Dell computer (with a refresh rate of $85 \mathrm{~Hz}$ and a resolution of 1024 by 768) running E-Prime 2.0 software (Psychology Software Tools, Inc. Pittsburgh, PA). Participants were instructed to seat in a soundproof chamber at a distance of approximately $60 \mathrm{~cm}$ away from the screen and to complete the error awareness task. The stimuli were six colored Chinese characters [green $(0,255,0)$, red $(255,0,0)$, yellow $(255,255,0)$, blue $(0,0,255)$, purple $(255,0,255)$, and white $(255,255,255)]$, which were presented on a black background. Participants were asked to respond to each of the words with a single button press as quickly and correctly as possible when font color of the word and its semantic content were consistent (go trials), and to withhold their responses in the incongruent No-go and repeat No-go trials (No-go trials, which have been introduced in the result section). The stimulus-response mappings were counterbalance across participants. For half of the participants, go trials were mapped to "A" key (left index finger), the subjective rating of correct responses were mapped to " $K$ " key (right index finger), and the subjective rating of error responses were mapped to "L" key (right middle finger). For the other half of participants, go trials were mapped to "L" key (right index finger), the subjective rating of correct responses were mapped to "A" key (left middle finger), and the subjective rating of error responses were mapped to "S" key (left index finger).

Before experiment, participants first completed a practice block of 30 trials to be familiar with response rules. Then, they completed 6 experiment blocks of 210 trials, with a one-minute break between blocks. For each block, 30 No-go trials pseudo-randomly arranged throughout the serial presentation of 180 go trials. Moreover, the proportion of repeat No-go and incongruent No-go trials was equal in each block.

Experimental procedure. Figure 1 displayed the schematic of error awareness task. On each trial, a white fixation cross (+) was presented for $200 \mathrm{~ms}$ followed by a $300 \mathrm{~ms}$ blank screen. The stimulus was then presented on the central of the screen for a maximum of $800 \mathrm{~ms}$ (terminated after go press within this interval). After the stimulus disappearance, the screen remained black for $1,000 \mathrm{~ms}$. Next, the hash (\#) cue reminded participant to rate his response accuracy (error or correct). The hash cue was terminated by a key press within 1,000 ms, followed by an inter-trial interval of $600 \mathrm{~ms}$.

EEG data acquisition. The EEG data were recorded using a 64-channel Brain Products system (Brain Products $\mathrm{GmbH}$, Germany; passband: $0.01-100 \mathrm{~Hz}$; sampling rate: $500 \mathrm{~Hz}$ ) that was connected to a standard EEG cap based on the extended 10-20 system. All signals were on-line referenced to electrode FCz and off-line algebraic re-reference to the average of the left and right mastoids. Electrode FCz was re-instated ${ }^{42,43}$. The vertical electrooculogram (EOG) was recorded from electrode located below the right eye. The horizontal EOG was recorded from electrode located at the outer canthus of the right eye. Inter-electrode impedance was maintained below $5 \mathrm{k} \Omega$. In the traditional ERP analysis, data were filtered offline with a passband $0.1-30 \mathrm{~Hz}(12 \mathrm{~dB} /$ oct). Additionally, to examine whether ERN was phase-locked in the theta band, the ERN was analyzed with a passband $4-8 \mathrm{~Hz}(12 \mathrm{~dB} /$ oct, theta-ERN), $8-14 \mathrm{~Hz}(12 \mathrm{~dB} /$ oct, alpha-ERN) and $14-30 \mathrm{~Hz}(12 \mathrm{~dB} /$ oct, beta-ERN), respectively. The correction of ocular artifacts was conducted by Independent Component Analysis (ICA) in Brain Vision Analyzer 2.0 (Brain Products GmbH, Germany). 64 ICA components were identified for each participant and IC scalp topographies, time courses, and spectral characteristics were inspected visually to identify and reject components related to blinks and eye-movements ${ }^{44}$. Moreover, trials in which EEG voltages exceeded a threshold of $\pm 100 \mu \mathrm{V}$ during the recording epoch were excluded from averaging.

ERP analysis. EEG data were preprocessed by Brain Vision Analyzer 2.0. Then, the resulting data were segmented and time locked to the onset of response (200 ms pre- and $800 \mathrm{~ms}$ post-response). These epochs were baseline-corrected relative to the interval -200 to $-100 \mathrm{~ms}^{25}$ and were averaged separately for correct go responses, aware errors and unaware errors. The ERN was defined as the most negative peak in the -20 to $50 \mathrm{~ms}$ time window over fronto-central region [Fig. $2 \mathrm{a}$; $(\mathrm{FCz}+\mathrm{FC} 1+\mathrm{FC} 2+\mathrm{Cz}+\mathrm{C} 1+\mathrm{C} 2) / 6]$. And the Pe was defined based on the mean amplitude in the time window of 150 to $500 \mathrm{~ms}$ over parietal region [Fig. $2 \mathrm{~b} ;(\mathrm{CPz}+\mathrm{CP} 1+\mathrm{C}$ $\mathrm{P} 2+\mathrm{Pz}+\mathrm{P} 1+\mathrm{P} 2) / 6]$.

Time-frequency analysis. The preprocessing of time-frequency analysis was conducted by Brain Vision Analyzer 2.0 and EEGLAB (an open source toolbox running in the MATLAB environment for EEG signal processing $)^{45,46}$. Considering the relatively low time resolution for the time-frequency analysis, we chose a relatively long baseline to get a steady estimation for the low frequencies. Thus, we segmented EEG data into a time window from -600 to $800 \mathrm{~ms}$ that was time-locked to the onset of response and corrected the baseline using the interval of pre-response -600 to $-100 \mathrm{~ms}$. After the baseline correction was accomplished in the Analyzer 2.0, data were imported into EEGLAB. Remaining artifacts in the EEG were addressed using a $\pm 100 \mu \mathrm{V}$ threshold by EEGLAB, and corresponding epochs were excluded.

After all EEG data were reprocessed, oscillatory power (time-frequency representation) was obtained from single trial EEG epochs using the continuous Morlet wavelet transform (CWT) conducted by Letswave sofware (http://amouraux.webnode.com $)^{47}$. The parameters of central frequency $(\omega)$ and restriction $(\sigma)$ in CWT were 5 and 0.15 respectively, and time-frequency representations were explored from 1 to $30 \mathrm{~Hz}$ in steps of $0.58 \mathrm{~Hz}^{48}$. Then, single trial time-frequency representations were averaged to obtain averaged time-frequency representation. Subsequently, to identify the modulations of ongoing EEG rhythms, an event-related spectral perturbation (ERSP) was calculated for every time-frequency pixel in the averaged time-frequency representation. For 
each estimated frequency, ERSP was shown as a transient increase or decrease in oscillatory power and was baseline-corrected according to the following formula: $E R_{t, f} \%=\left[A_{t, f}-R_{f}\right] / R_{f}$, where $A_{t, f}$ was the signal power at a given time $(t)$ and frequency $(f)$, and $R_{f}$ was the signal power averaged within the baseline interval ${ }^{49}$. To avoid edge artifacts when performing CWT, pre-response time interval -550 to $-150 \mathrm{~ms}$ was used as the baseline interval in the time-frequency analysis.

When the original power was transformed to ERSP in the time-frequency representations, an exploratory data-driven approach was performed to identify the spatial regions of interest (S-ROIs) and time-frequency regions of interest (TF-ROIs). The exploratory data-driven analysis routine was performed as follows.

Firstly, several TF-ROIs associated with error awareness processing were roughly identified by calculating the time-frequency difference map corresponding to aware and unaware errors across all electrodes. Secondly, based on the defined TF-ROIs (such as theta and alpha), the mean of time-frequency pixels in a specific TF-ROI was calculated respectively for aware and unaware errors, and the results corresponding to the electrodes were plotted as scalp maps. According to the difference map between aware errors and unaware errors, fronto-central region $[(\mathrm{Fz}+\mathrm{FCz}+\mathrm{Cz}) / 3]$ and occipito-parietal region $[(\mathrm{Pz}+\mathrm{P} 3+\mathrm{P} 4+\mathrm{POz}+\mathrm{PO} 3+\mathrm{PO} 4) / 6]$ were identified as the S-ROIs (Fig. 4b). Thirdly, based on the defined S-ROIs, time-frequency representation of the ERSP magnitude difference between aware and unaware errors was calculated. And then, the resulting ERSP magnitudes in the post-response interval were further examined whether and when differed from the ERSP magnitudes in the pre-response interval utilizing a boot-strapping method ${ }^{50}$. According to the $p$ map (FDR corrected) between aware and unaware errors, the maximal time-frequency power and corresponding peak power latencies were chosen as TF-ROI. Since TF-ROI had to be composed of more than 75 consecutive significant time points $(>150 \mathrm{~ms})^{51}$. Moreover, frequencies below $4 \mathrm{~Hz}$ were not considered for oscillations because such an extremely low frequency band is often subject to artifacts due to sweating, movement and electrode drift ${ }^{52}$. In this case, theta (4-7 Hz, -150 to $200 \mathrm{~ms}$ ) and alpha $(8-14 \mathrm{~Hz}, 200$ to $600 \mathrm{~ms}$ ) bands were chosen as the TF-ROIs (Fig. $4 \mathrm{a}$ ).

Notably, the above analyses in the time-frequency domain showed the total activity based on the signal of single error trial, including phase-locked and non-phase-locked components. To make clear the functional role of phase-locked component in the error awareness processing, we also computed the phase-locked activity for each condition, electrode and participant. The analyses on phase-locked time frequency based on the average signal of each error type (aware and unaware errors). The average signal of each error type was the time- and phase-locked neural activities elicited by events of interest. Then, the time-frequency representation was obtained from average signal of each error type using CWT conducted by Letswave software. Methodology on the calculation of ERSP and the definition of S-ROI and TF-ROI in the analysis of phase-locked time frequency was similar to the time-frequency analysis of total activity. As a result, fronto-central region $[(\mathrm{Fz}+\mathrm{FCz}+\mathrm{Cz}) / 3]$ was chosen as the $\mathrm{S}$-ROI and theta band (4-7 Hz, -150 to $200 \mathrm{~ms}$ ) was chosen as the TF-ROI (Fig. 5).

\section{Data availability}

The datasets generated during and/or analysed during the current study are available from the corresponding author on reasonable request.

Received: 28 November 2019; Accepted: 11 February 2020;

Published online: 04 March 2020

\section{References}

1. Falkenstein, M., Hohnsbein, J., Hoormann, J. \& Blanke, L. Effects of crossmodal divided attention on late ERP components. II. Error processing in choice reaction tasks. Electroencephalography and Clinical Neurophysiology 78, 447-455 (1991).

2. Gehring, W. J., Goss, B., Coles, M. G. H., Meyer, D. E. \& Donchin, E. A neural system for error detection and compensation. Psychological Science 4, 385-390 (1993).

3. Overbeek, T., Nieuwenhuis, S. \& Ridderinkhof, K. Dissociable components of error processing: On the Functional Significance of the Pe Vis-à-vis the ERN/Ne. Journal of Psychophysiology 19, 319-329 (2005).

4. Debener, S. et al. Trial-by-trial coupling of concurrent electroencephalogram and functional magnetic resonance imaging identifies the dynamics of performance monitoring. The Journal of Neuroscience 25, 11730-11737 (2005).

5. Iannaccone, R. et al. Conflict monitoring and error processing: New insights from simultaneous EEG-fMRI. Neuroimage 105, 395-407 (2015).

6. Orr, C. \& Hester, R. Error-related anterior cingulate cortex activity and the prediction of conscious error awareness. Frontiers in human neuroscience 6 (2012).

7. Di Gregorio, F., Steinhauser, M. \& Maier, M. E. Error-related brain activity and error awareness in an error classification paradigm. Neuroimage 139, 202-210 (2016).

8. Logan, D. M., Hill, K. R. \& Larson, M. J. Cognitive control of conscious error awareness: error awareness and error positivity $(\mathrm{Pe})$ amplitude in moderate-to-severe traumatic brain injury (TBI). Frontiers in Human. Neuroscience 9, 397 (2015).

9. Shalgi, S., Barkan, I. \& Deouell, L. Y. On the positive side of error processing: error-awareness positivity revisited. European Journal of Neuroscience 29, 1522-1532 (2009).

10. Wessel, J. R., Danielmeier, C. \& Ullsperger, M. Error awareness revisited: accumulation of multimodal evidence from central and autonomic nervous systems. Journal of cognitive neuroscience 23, 3021-3036 (2011).

11. Maier, M., Steinhauser, M. \& Hübner, R. Is the error-related negativity amplitude related to error detectability? Evidence from effects of different error types. Journal of Cognitive Neuroscience 20, 2263-2273 (2008).

12. Navarro-Cebrian, A., Knight, R. T. \& Kayser, A. S. Error-monitoring and post-error compensations: dissociation between perceptual failures and motor errors with and without awareness. The Journal of Neuroscience 33, 12375-12383 (2013).

13. Dhar, M. \& Pourtois, G. Early error detection is generic, but subsequent adaption to errors is not: evidence from ERPs. Neuropsychologia 49, 1236-1245 (2011).

14. Endrass, T., Franke, C. \& Kathmann, N. Error awareness in a saccade countermanding task. Journal of Psychophysiology 19, 275-280 (2005).

15. O'Connell, R. G. et al. The role of cingulate cortex in the detection of errors with and without awareness: a high-density electrical mapping study. European Journal of Neuroscience 25, 2571-2579 (2007). 
16. Coles, M. G., Scheffers, M. K. \& Holroyd, C. B. Why is there an ERN/Ne on correct trials? Response representations, stimulusrelated components, and the theory of error-processing. Biological psychology 56, 173-189 (2001).

17. Botvinick, M. M., Braver, T. S., Barch, D. M., Carter, C. S. \& Cohen, J. D. Conflict monitoring and cognitive control. Psychological Review 108, 624-652 (2001).

18. Yeung, N., Botvinick, M. M. \& Cohen, J. D. The neural basis of error detection: conflict monitoring and the error-related negativity. Psychological review 111, 931 (2004).

19. Hughes, G. \& Yeung, N. Dissociable correlates of response conflict and error awareness in error-related brain activity. Neuropsychologia 49, 405-415 (2011).

20. Luu, P., Tucker, D. M. \& Makeig, S. Frontal midline theta and the error-related negativity: neurophysiological mechanisms of action regulation. Clinical Neurophysiology 115, 1821-1835 (2004).

21. Munneke, G.-J., Nap, T. S., Schippers, E. E. \& Cohen, M. X. A statistical comparison of EEG time-and time-frequency domain representations of error processing. Brain research 1618, 222-230 (2015).

22. Murphy, P. R., Robertson, I. H., Harty, S. \& O'Connell, R. G. Neural evidence accumulation persists after choice to inform metacognitive judgments. eLife, e11946 (2015).

23. Hester, R., Foxe, J. J., Molholm, S., Shpaner, M. \& Garavan, H. Neural mechanisms involved in error processing: a comparison of errors made with and without awareness. Neuroimage 27, 602-608 (2005)

24. Wessel, J. R. Error awareness and the error-related negativity: evaluating the first decade of evidence. Frontiers in human neuroscience 6 (2012).

25. Endrass, T., Reuter, B. \& Kathmann, N. ERP correlates of conscious error recognition: aware and unaware errors in an antisaccade task. European Journal of Neuroscience 26, 1714-1720 (2007).

26. Hoonakker, M., Doignoncamus, N. \& Bonnefond, A. Performance monitoring mechanisms activated before and after a response: a comparison of aware and unaware errors. Biological Psychology 120, 53-60 (2016).

27. Olvet, D. M. \& Hajcak, G. The stability of error-related brain activity with increasing trials. Psychophysiology 46, 957-961 (2009).

28. Wang, L., Tan, J., Chen, J. \& Chen, A. The influence of observers' sex on attention-demanding performance depends on performers' sex. Frontiers in Psychology 6 (2015).

29. Cavanagh, J. F. \& Frank, M. J. Frontal theta as a mechanism for cognitive control. Trends in cognitive sciences 18, 414-421 (2014).

30. Cavanagh, J. F., Meyer, A. \& Hajcak, G. Error-Specific Cognitive Control Alterations in Generalized Anxiety Disorder. Biological Psychiatry Cognitive Neuroscience \& Neuroimaging 2, 413 (2017).

31. Marco-Pallares, J., Camara, E., Munte, T. F. \& Rodriguez-Fornells, A. Neural mechanisms underlying adaptive actions after slips. Journal of Cognitive Neuroscience 20, 1595-1610 (2008).

32. Swann, N. et al. Deep brain stimulation of the subthalamic nucleus alters the cortical profile of response inhibition in the beta frequency band: a scalp EEG study in Parkinson's disease. Journal of Neuroscience the Official Journal of the Society for Neuroscience 31, 5721 (2011).

33. Trujillo, L. T. \& Allen, J. J. Theta EEG dynamics of the error-related negativity. Clinical Neurophysiology 118, 645-668 (2007).

34. Cohen, M. X. \& Van, G. S. Subthreshold muscle twitches dissociate oscillatory neural signatures of conflicts from errors. Neuroimage 86, 503-513 (2014).

35. Steinhauser, M. \& Yeung, N. Decision processes in human performance monitoring. The Journal of neuroscience 30, 15643-15653 (2010).

36. Steinhauser, M. \& Yeung, N. Error awareness as evidence accumulation: effects of speed-accuracy trade-off on error signaling. Frontiers in human neuroscience 6 (2012).

37. Cohen, M. X., Simon van Gaal, K. \& Lamme, V. A. Unconscious errors enhance prefrontal-occipital oscillatory synchrony. Frontiers in human neuroscience 3 (2009).

38. Cavanagh, J. F. et al. Subthalamic nucleus stimulation reverses mediofrontal influence over decision threshold. Nature neuroscience 14, $1462(2011)$.

39. Di Gregorio, F., Maier, M. E. \& Steinhauser, M. Errors can elicit an error positivity in the absence of an error negativity: Evidence for independent systems of human error monitoring. Neuroimage 172, 427-436 (2018).

40. Maier, M. E. \& Steinhauser, M. Working memory load impairs the evaluation of behavioral errors in the medial frontal cortex. Psychophysiology 54, 1472-1482 (2017).

41. Fischer, A. G., Klein, T. A. \& Ullsperger, M. Comparing the error-related negativity across groups: The impact of error-and trialnumber differences. Psychophysiology 54, 998-1009 (2017).

42. Zendel, B. R. \& Alain, C. Enhanced attention-dependent activity in the auditory cortex of older musicians. Neurobiology of Aging 35, 55 (2014).

43. Zhu, X., Wu, H., Yang, S. \& Gu, R. The influence of self-construal type on outcome evaluation: Evidence from event-related potentials. International Journal of Psychophysiology Official Journal of the International Organization of Psychophysiology 112, 64-69 (2017).

44. Navarro-Cebrian, A., Knight, R. T. \& Kayser, A. S. Frontal monitoring and parietal evidence: mechanisms of error correction. Journal of cognitive neuroscience 28, 1166-1177 (2016).

45. Makeig, S., Debener, S., Onton, J. \& Delorme, A. Mining event-related brain dynamics. Trends in cognitive sciences 8, 204-210 (2004).

46. Wang, L. et al. Disentangling the impacts of outcome valence and outcome frequency on the post-error slowing. Scientific reports 5 (2015).

47. Mouraux, A. \& Iannetti, G. Across-trial averaging of event-related EEG responses and beyond. Magnetic resonance imaging 26, 1041-1054 (2008).

48. Zhao, Y. et al. Concurrent working memory task decreases the Stroop interference effect as indexed by the decreased theta oscillations. Neuroscience 262, 92-106 (2014).

49. Pfurtscheller, G. \& Lopes da Silva, F. Event-related EEG/MEG synchronization and desynchronization: basic principles. Clinical neurophysiology 110, 1842-1857 (1999).

50. Delorme, A. \& Makeig, S. EEGLAB: an open source toolbox for analysis of single-trial EEG dynamics including independent component analysis. Journal of neuroscience methods 134, 9-21 (2004).

51. Hu, L., Zhao, C., Li, H. \& Valentini, E. Mismatch responses evoked by nociceptive stimuli. Psychophysiology 50, 158-173 (2013).

52. He, B. J. \& Raichle, M. E. The fMRI signal, slow cortical potential and consciousness. Trends in cognitive sciences 13, 302-309 (2009).

\section{Acknowledgements}

We thank Professor Li Hu (University of Chinese Academy of Sciences) for his generous suggestions in data analysis and Doctor Zhenzhen Xu (Vrije University Amsterdam) for polishing the language.

\section{Author contributions}

L.J.W. and A.T.C. conceived and designed the experiment. L.J.W. and Y.G. performed the experiment and analyzed EEG recordings. L.J.W. and A.T.C. wrote the manuscript. G.X.Z. contributed to refine the language. 


\section{Competing interests}

Prof. Chen and Dr. Wang have been funded by National Natural Science Foundation of China (61431013, 31771254). Dr. Wang received further support from Postdoctoral Research Grant in Henan Province (001802013). Prof. Zhao and Dr. Gu declare no potential conflict of interest.

\section{Additional information}

Correspondence and requests for materials should be addressed to A.C.

Reprints and permissions information is available at www.nature.com/reprints.

Publisher's note Springer Nature remains neutral with regard to jurisdictional claims in published maps and institutional affiliations.

(c) (i) Open Access This article is licensed under a Creative Commons Attribution 4.0 International License, which permits use, sharing, adaptation, distribution and reproduction in any medium or format, as long as you give appropriate credit to the original author(s) and the source, provide a link to the Creative Commons license, and indicate if changes were made. The images or other third party material in this article are included in the article's Creative Commons license, unless indicated otherwise in a credit line to the material. If material is not included in the article's Creative Commons license and your intended use is not permitted by statutory regulation or exceeds the permitted use, you will need to obtain permission directly from the copyright holder. To view a copy of this license, visit http://creativecommons.org/licenses/by/4.0/.

(c) The Author(s) 2020 